\title{
TCOM \\ Naming is framing: the effects of a technological name on the interpretation of a technology
}

\section{Reginald Boersma, P. Marijn Poorvliet and Bart Gremmen}

\begin{abstract}
In this article, we follow up on food scientists' findings that people judge new food technologies and related products (un)favourably immediately after just hearing the name of the technology. From the reactions, it appears that people use their attitudes to technologies they know to evaluate new technologies. Using categorization theory, in this study we have found that, by triggering associations with a familiar technology, a name of the new technology can be enough to determine emerging attitudes. Comparison between the technology used for categorization and another familiar technology had a slight influence on the attitude formation process.
\end{abstract}

Keywords

DOI

Public perception of science and technology; Public understanding of science and technology

https://doi.org/10.22323/2.18060204

Submitted: 13th December 2018

Accepted: 8th November 2019

Published: 9th December 2019

Introduction

In modern society, people do not have time to become engaged with all scientific advancements they encounter. Science communication is oftentimes limited to communicating just a scientific name. When a name is all the information people have, the associations attached to this name can have far-reaching consequences; research focusing on the impact of scientific names is, however, largely lacking. The current paper, we focus on the consequences of the chosen name of a new food production technology on public attitudes. We do so by a novel approach in which we do not provide information about a technology, but rather investigate how people use their associations to give meaning to abstract technological terms. The key question is whether a name is enough to systematically influence emerging attitudes.

When a new technology is developed, the name of the technology is hardly ever questioned. Yet, there are reports from technical experts about people rejecting genomics-assisted breeding after hearing the name genomics. For example, scientists involved in genomics accelerated breeding report that, when confronted with the name 'genomics,' people who do not have any knowledge about genomics reject related developments for reasons that apply to genetic manipulation (GM) 
rather than genomics [Hall, 2010]. In Van Dam and De Vriend's [2002] report about public perceptions of genomics, the researchers believe that people use their knowledge about GM to answer questions about genomics. Scientists involved in nanotechnology experience that consumers tend to reject nanotechnology in general and foods produced with nanotechnology because consumers are especially familiar with harmful nanoparticles and therefore believe that all nanotechnologies are dangerous [Kampers, 2009]. Other examples where names triggered similar unexpected responses because a lack of related theoretical knowledge by the public are 'cultured' and 'in-vitro meat' [Friedrich, 2016] and genetic manipulation itself, [Bauer, Durant and Gaskell, 1998; Hansen, 2010; see also Ingold and Kurttila, 2000, on similar issues caused by the term climate].

From the above reports, a pattern emerges where people use knowledge about a technology they know, and which appears related in name, to respond to an unfamiliar food production technology. By using the knowledge and attitudes relating to the familiar concepts, they can in turn respond to the unfamiliar concepts [Boersma and Gremmen, 2018; - see also Brunel et al., 2017, for an example how reactions towards nanotechnology may be anchored in GMO knowledge], even if the re-used knowledge doesn't apply to the new technology. This may lead to misconstrued expectations, as illustrated by the case of genomics. ${ }^{1}$ Genomics can be used to create new fruit and vegetable cultivars through natural sexual reproduction. When new cultivars are created using GM, artificial recombination of genes is applied, which makes the technology highly controversial. When we compare genomics with other techniques, genomics actually applies traditional breeding (TB), i.e. sexual reproduction, when creating new crops. The difference between genomics and TB is that genomic scanning is applied after reproduction to check whether desired genes are transferred in the breeding process. From the perspective of reproduction, genomics is better understood when people apply their feelings and beliefs about TB rather than about GM. Ironically, it appears that, because of the name, they actually link genomics with GM when evaluating the technology.

When this occurs, people will not only make misconstrued consumer evaluations and decisions, the initial impressions can also have a significant impact when interactions between the experts and lay do occur. As recalled by Hall [2010], a meeting to explore possible collaboration in developing genomics with organic farmers -who did not object to the principles of genomics- was lost because of their initial impressions about genomics. After hearing the name, they assumed it was similar to GM, and it took an entire meeting to correct the idea. Having similar experiences communicating the technological nature and virtues of in-virto meat, Friedrich [2016] proposed finding a new name for the technology as, according to Friedrich, "First impressions are critical. We don't want to start a discussion by having to disabuse people of ... inaccurate assumptions".

To prevent these inaccurate assumptions, it is important to understand the role of the language and names used by experts in shaping the impressions by lay. In the

\footnotetext{
${ }^{1}$ In this research, the term genomics is used in the same way as it is used by plant breeders. Although the term genomics is generally referring to study of genomes, it is also the standard term plant breeders use to replace the full name genomics accelerated breeding when describing genomics applied to plant breeding in communication both among themselves and to the public [see Van Dam and De Vriend, 2002].
} 
current paper, we test the idea that people use a familiar food production technology that appears related by name to give meaning to a technology with which they are unfamiliar. Using an experiment, we systematically investigate whether or not the process takes place and what the consequences are for emerging attitudes about the unfamiliar concept. We build on the observation that people possibly use knowledge about GM to respond to questions about genomics, and we use genomics as an example of the transfer of meaning from one concept to another.

A novelty in the current approach is that we focus on how people evaluate a technology without providing any further information than the name. Instead of focussing on how people respond to information, the current research tries to understand the behaviours of the uninformed. The key concern is how people deal with a lack of information when they ask themselves the question 'What is it?' after hearing the name of an unfamiliar technology.

Two theories in social and cognitive psychology relate in particular to the expected process. Using these theories, we formulate and test hypotheses about how they influence the perception of a technology. The first theory we investigate is categorization theory [Loken, Barsalou and Joiner, 2008; Rosch, 1978], which describes the way people use knowledge about familiar concepts to give meaning to unfamiliar concepts when trying to answer a 'what is it' question when faced with a new concept. The theory thus offers an explanation as to why people use their knowledge about GM to evaluate genomics. Second, we turn our attention to comparison effects. Comparison effects relate to categorization theory because knowledge about familiar concepts can be used in different ways to give meaning to unfamiliar concepts [Gregan-Paxton, Hoeffler and Zhao, 2005; Herr, 1986; Higgins, 1989; Stoner, Loken and Stadler Blank, 2018].

\section{Categorization theory}

A theory widely applied in consumer behaviour to predict the way people react to new products is categorization theory [Loken, Barsalou and Joiner, 2008]. According to the theory, people organize their knowledge about the world in clusters of related concepts. For example, a person might have a mental category of cats, computers and tomatoes. Knowledge about these concepts is linked not so much to the members of the category as to the category itself. For example, instead of remembering that each tomato we encounter is red, we remember that tomatoes are usually red.

Although categorization theory mainly describes the way we store and organize knowledge, it can also provide insight into the way we try to give meaning to new concepts [Gregan-Paxton and John, 1997]. According to the theory, we try to understand new concepts by finding familiar concepts that are, in some way, similar. For example, when a person encounters a cherry tomato for the first time, this person might judge it to be a special kind of tomato and store the new concept in the category tomatoes. This process is called categorization. After categorization, the person can use knowledge about tomatoes to give meaning to, and make decisions about, the new cherry tomato without being fully educated on the subject.

Additionally, when attitudes are activated, they might be applied to the new category member alongside knowledge. For example, if a person has negative 
feelings towards tomatoes, the new cherry tomato will probably be evaluated negatively. This is called attitude extension [Muthukrishnan and Weitz, 1991] and can be described as the extension of an existing attitude about familiar concepts towards a new category member. It is important to note that attitude extension does not require any knowledge about the new concept itself. Therefore, attitude extension provides a quick way to reach an evaluation without getting to know the new construct. The emerging attitudes are the result of what people believe the new concept is related to, rather than an evaluation of the concept itself.

It has been found that, when people have little knowledge about a subject, the categorization of new, related concepts is strongly influenced by superficial, non-functional attributes of the new concept rather than functional attributes [Gregan-Paxton, 2001; Gregan-Paxton and John, 1997]. We argue that the name can be an important superficial attribute of an unknown concept. A name can act as a conceptual label that guides people to known concepts that can be used to give meaning to the new concept [Gregan-Paxton, Hoeffler and Zhao, 2005]. Therefore, it can be very determinant in the selection of the category which will be used for categorization. When people are confronted with the name of an invisible technology of which they have no further knowledge, the only attribute available to them for categorization is the name. For example, when people are confronted with the genomics concept, there is nothing to be studied except for the name. Therefore, especially when people have little knowledge about gene-technologies, the name genomics might quickly be associated with genetic manipulation.

Categorization theory can provide an explanation for the way people make decisions and form initial attitudes about genomics. In the current study, we test the hypothesis that, when people do not have any information about genomics, they use attitude extension from genetic manipulation to form an attitude about genomics because of the similarity in name.

Hypothesis 1a: People use their knowledge and attitudes about genetic manipulation to form a response about genomics.

If the name genomics leads to categorization with genetic manipulation, then another name would have the potential to lead to categorization with other technologies. As mentioned earlier, in genomics, the step in which traits are combined entails natural sexual reproduction. A name stressing this particular component of genomics, for example the (fictional) name 'natural crossing' could, with respect to activating related knowledge, lead to a more appropriate categorization from a technical perspective and consequently a more favourable evaluation of the technology. Therefore, we study the response of respondents to the fictional name, natural crossing.

Hypotheses 1b: People use their knowledge and attitudes about traditional breeding to form a response towards natural crossing.

\section{Comparison effects}

Categorization plays an important role in the way attitudes are influenced by the context, not only through the categorization itself, but also by the way 
activated categories are used for evaluation [Gregan-Paxton, 2001; Gregan-Paxton, Hoeffler and Zhao, 2005; Herr, 1986]. Especially when people experience ambiguity towards a concept, attitudes can be influenced by the context in which it is reported [Higgins, 1989]. Therefore, attitudes formed and reported can be influenced by the context, particularly in a situation where people try to give meaning to an abstract technology, making the interpretation of genomics susceptible to comparison effects.

When a concept under evaluation is placed within a category, information linked to the category can act as an interpretation frame that can be used to give meaning to the unfamiliar concept [Gregan-Paxton, 2001; Gregan-Paxton, Hoeffler and Zhao, 2005; Higgins, 1989]. When the presentation context in which the new concept is presented is used for categorization, then the evaluation shifts towards the evaluation of the presentation context. This process is called assimilation. For example, if genomics is presented in a context with GM, and people believe the two are the same, the evaluation of genomics will shift towards the evaluation of GM because the context reminds them of GM attributes, which can, in turn, be used to evaluate genomics.

When a concept is presented in a presentation context which is not regarded as suitable to give meaning to an unfamiliar concept, the presentation context can act as a standard for comparison [Herr, 1986; Mussweiler, Michels and Weiss, 2017]. For example, when genomics is presented in a context with traditional breeding and it is believed that genomics is a form of GM because of categorization by name, people will be inclined to compare genomics with the context. Rather than using the context to give meaning by focusing on (what are believed to be) shared attributes, the people will focus on the presumed differences. Consequently, genomics might be evaluated even more negatively because the evaluator focuses on favourable attributes of TB, which genomics is believed not to have. In addition, when the presentation context is experienced as potentially inappropriate, people might correct their evaluation away from the context to compensate for the influence.

Judged on its technical attributes, genomics might be explained best by comparing it with traditional breeding. Unfortunately, when people already believe that genomics is GM, comparison effects might result in even more unfavourable attitudes if they make this comparison. In the current experiment, we test the hypotheses that the name genomics will lead to an even more negative evaluation when it is combined with the TB concept.

Hypotheses 2a: The name genomics will lead to a more negative evaluation when presented in combination with traditional breeding than when it is presented with GM.

In the current line of reasoning, the name natural crossing can be expected to have an opposite effect. If natural crossing makes people believe that the new technology is related to $\mathrm{TB}$, then it can be expected that the evaluation will assimilate towards TB and contrast away from the evaluation of GM.

Hypotheses 2b: The name natural crossing will lead to a more favourable evaluation when presented in combination with GM than when it is presented with TB. 
- Hypotheses: In situations where people have to evaluate an unfamiliar technology they use their knowledge about a familiar technology that appears similar by name. These evaluations are also influenced by comparing the evaluations with other known technologies

- 120 Participants participated in a 2 context (GM/Traditional breeding) $\times 2$ unfamiliar technology (genomics/natural crossing) design experiment.

- Results show participant use the names of the technology to achieve evaluations through categorizing the unfamiliar technology with a familiar method

In total, 120 students from (....) participated and received a nominal five euros in compensation. The experiment had a 2 (context: genetic manipulation versus traditional breeding) $\times 2$ (name unfamiliar technology: genomics versus natural crossing) design, and participants were randomly distributed.

\section{Procedure}

Introduction and manipulation. On entry, participants were welcomed and randomly assigned to a computer. For experimental purposes (see need for closure measurements), they were told that they would participate in a series of experiments and asked to sign a consent form informing them that the results would be processed anonymously and that they could stop at any time if they wished to do so. The experiment began with an introduction presenting a cover story that the aim of the research was to find out people's thoughts and opinions about different ways of making new kinds of fruits and vegetables. The context manipulation then followed, in which a way of making a new cultivar was explained.

Participants in the traditional breeding context read:

"In agriculture, new plant varieties are developed. One way to develop a new variety is traditional breeding. When traditional breeding is applied, pollen from one plant is put on the flower of another. The new plant that will result is a crossing of the 'parents,' and will share characteristics with both of them. For example, a plant bearing many tomatoes and a plant bearing round tomatoes can be crossed to produce a plant bearing many round tomatoes."

Participants in the genetic manipulation context where presented the following text:

"In agriculture, new plant varieties are developed. One way to develop a new variety is genetic manipulation. When genetic manipulation is applied, part of the DNA of one plant is put in the DNA of another. From the new DNA, a plant will develop containing characteristics of both plants. For example, the DNA of a plant 
bearing many tomatoes can be combined with the DNA of a plant bearing round tomatoes to produce a plant bearing many round tomatoes."

When the continue button was pressed, an extra line appeared on screen presenting the unfamiliar technology. For participants in the genomics context, the following text was added:

"There are more ways of developing new plant varieties. One of them is genomics."

Participants in the natural crossing context were told:

"There are more ways of developing new plant varieties. One of them is natural crossing."

Attitude measurements. After the manipulation, participants rated the unfamiliar context technology on 14 aspects on a 7-point scale adopted from Van den Heuvel et al. [2008]. Examples of aspects are the extent to which participants believed that the unfamiliar technology was useful ( $1=$ very useless, $7=$ very useful) and safe ( $1=$ very dangerous, 7 = very safe). In order to test the effects on the acceptance of food produced with the technology, the scale was extended with three questions about the actions towards a product produced with the technology and inquired about the extent to which the participant was willing to buy, eat and serve food produced with the unfamiliar technology $(1=$ absolutely not, $7=$ no problem with it), resulting in a total of 17 questions $(\alpha=.94)$. Participants were instructed to respond by giving their first impression, and to answer even if they did not know much about the technology. After the unfamiliar technology, the context technology was evaluated (genetic manipulation/traditional breeding) using the same questions $(\alpha=.96)$.

Categorization measurement. The categorization measurement followed these questions (see Figure 1) [Aron, Aron and Smollan, 1992]. Participants were confronted with seven pictures. Each picture contained a line on which two circles where placed, and the distance between the circles ranged from full overlap at the middle of the line to the maximum possible distance apart on the line. In one circle, the name of the unfamiliar context technology was presented, in the other, the name of the context technology. For example, participants in the GM/genomics group saw a picture of a circle with the name GM and a circle with the name genomics. Participants were asked to choose the picture that for them resembled best the way the two technologies where related to each other.

Control variable. The categorization measurements were followed by an open question, in which participants were asked to freely type what they knew about genomics (natural crossing). The answers to this question served as a check to determine whether or not participants where familiar with genomics.

In the current study, we were interested in the formation of emerging attitudes. To be able to investigate this development of emerging attitudes towards genomics, 


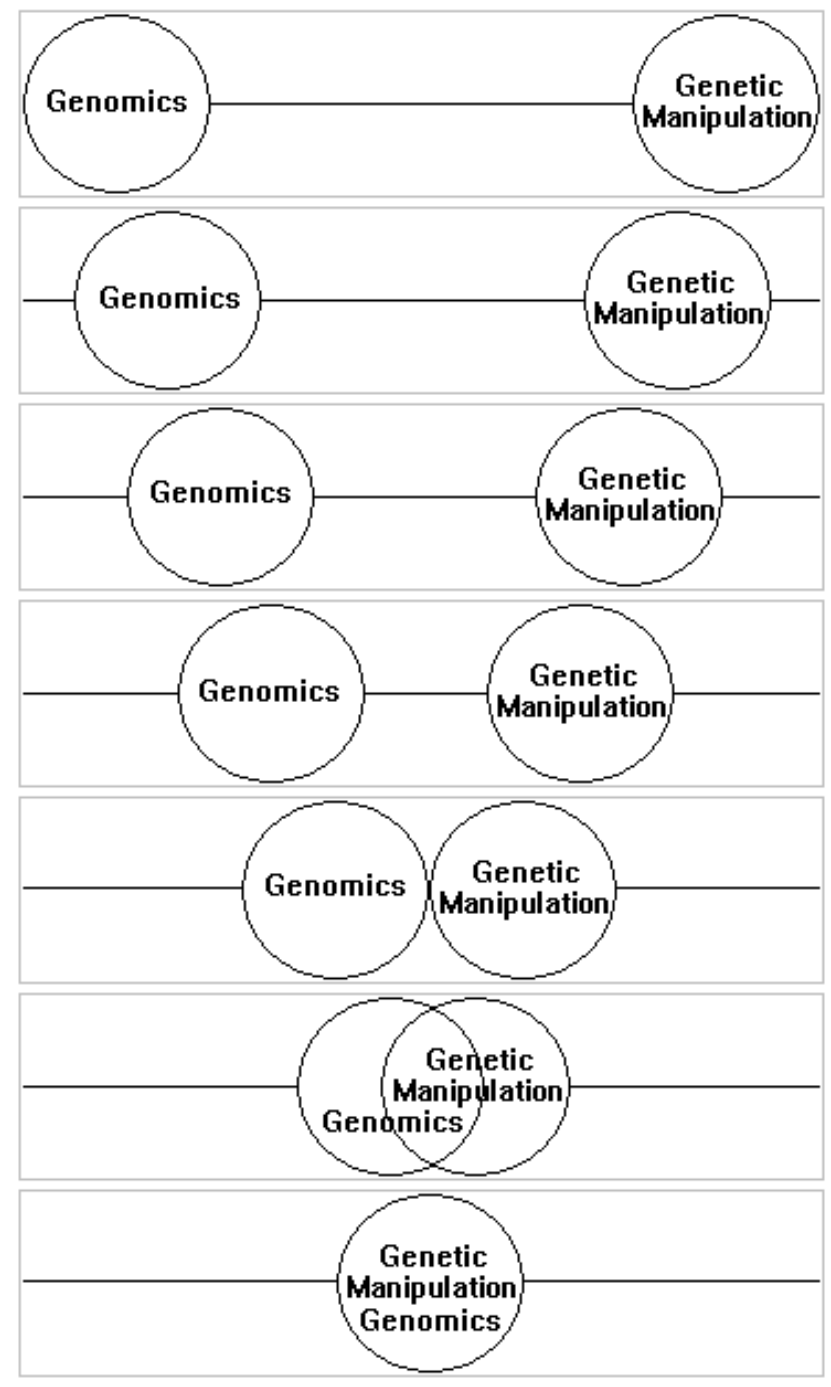

Totally different

The same

Figure 1. Measuring the perceived relation between genomics and genetic manipulation.

we needed participants who did not have well-established knowledge about, and stable attitudes towards, genomics. We used the responses to the question where participants were asked to write what they knew about genomics to check the extent to which they were familiar with the technology. In total, 58 participants were subject to the genomics context.

Of the 58 participants, one participant gave an answer showing knowledge about genomics, but the answer also included elements of GM. Because attributes of both technologies were used, it was not clear to what extent the difference was understood and therefore the participant remained included in the analyses. Of the remaining participants, three mentioned aspects of genomics, but expressed doubt about what the concept entails. These participants were also included because, by showing ambiguity, they met the criteria of not having clear knowledge. The remaining 54 participant did not show knowledge of genomics, with the result that all participants were included in the analyses. 
Using the graphical categorization question (Figure 1), we set out to determine the extent to which participants related the unfamiliar technology with the familiar technology about which they read an explanation. The expectation was that participants would believe genomics to be related to genetic modification and different from traditional breeding. When confronted with the name natural crossing, we expected participants to believe the concept to be related to traditional breeding and to be different than genetic manipulation. A 2 (context) $\times 2$ (name technology) ANOVA revealed a significant interaction effect, $F(1,116)=71.63, p<$ $.001, \eta_{\mathrm{p}}^{2}=.38$. Simple effect analyses revealed that, within the traditional breeding context, the distance between the context and natural crossing was perceived as smaller, $M=2.73, S D=0.83$, and the distance from genomics was perceived as larger, $M=4.38, S D=1.43, F(1,116)=26.46, p<.001$. In the genetic manipulation context, the opposite effect was observed. Here, the distance between genomics and the context was regarded as smaller, $M=2.69, S D=0.97$, and the distance between natural crossing and the context was perceived as larger, $M=4.84, S D=$ $1.53, F(1,116)=46.76, p<.001$. The results thus confirm our expectation. Genomics resulted in a strong association with genetic manipulation and not with traditional breeding. The name natural crossing resulted in the technology being closely related to traditional breeding and not to genetic manipulation.

With respect to attitude extension, the hypothesis was that the attitude towards familiar technologies would be used to form an initial attitude towards the unfamiliar technology. A scatter plot (see Figure 2) revealed the expected patterns, showing a clear relation between attitudes to genomics and GM and between natural crossing and TB. In line with Hypothesis 1a, linear regression revealed a significant correlation between the reported attitudes about genomics and genetic manipulation, $\mathrm{B}=.73,95 \% \mathrm{CI}[.62, .83], t(27)=14.16, p<.001$, with the attitudes to genetic manipulation predicting a large portion of the variance of the reported attitudes about genomics, $R^{2}=.88, F(1,27)=200.54, p<.001$. The result was similar for the attitudes about natural crossing and traditional breeding, $\mathrm{B}=.68,95 \% \mathrm{CI}$ $[.49, .87], t(28)=7.26, p<.001, R^{2}=.65, F(1,28)=52.78, p<.001$, which is in line with Hypothesis $1 \mathrm{~b}$. Following expectations, the results indicate that the attitude towards the unfamiliar technology is derived from the attitude towards the familiar technology with which people categorize the technology. There is a strong correlation between the attitudes towards genomics and the attitudes towards GM, and a strong correlation between the attitudes towards natural crossing and the attitudes towards TB.

The scatter plot does not show a relation between the evaluations of genomics and TB or natural crossing and GM. Linear regression did not reveal a relation between the attitudes about genomics and traditional breeding either, $\mathrm{B}=.18,95 \% \mathrm{CI}[-.26$, $.61], t(27)=.83, p=.42$, or between attitudes about natural crossing and genetic manipulation, $\mathrm{B}=.07,95 \% \mathrm{CI}[-.15, .29], t(30)=64, p=.53$. These results suggest that the relation between the attitude towards the unfamiliar technology and the context technology only exists for the combinations where the context is considered appropriate for categorization. 


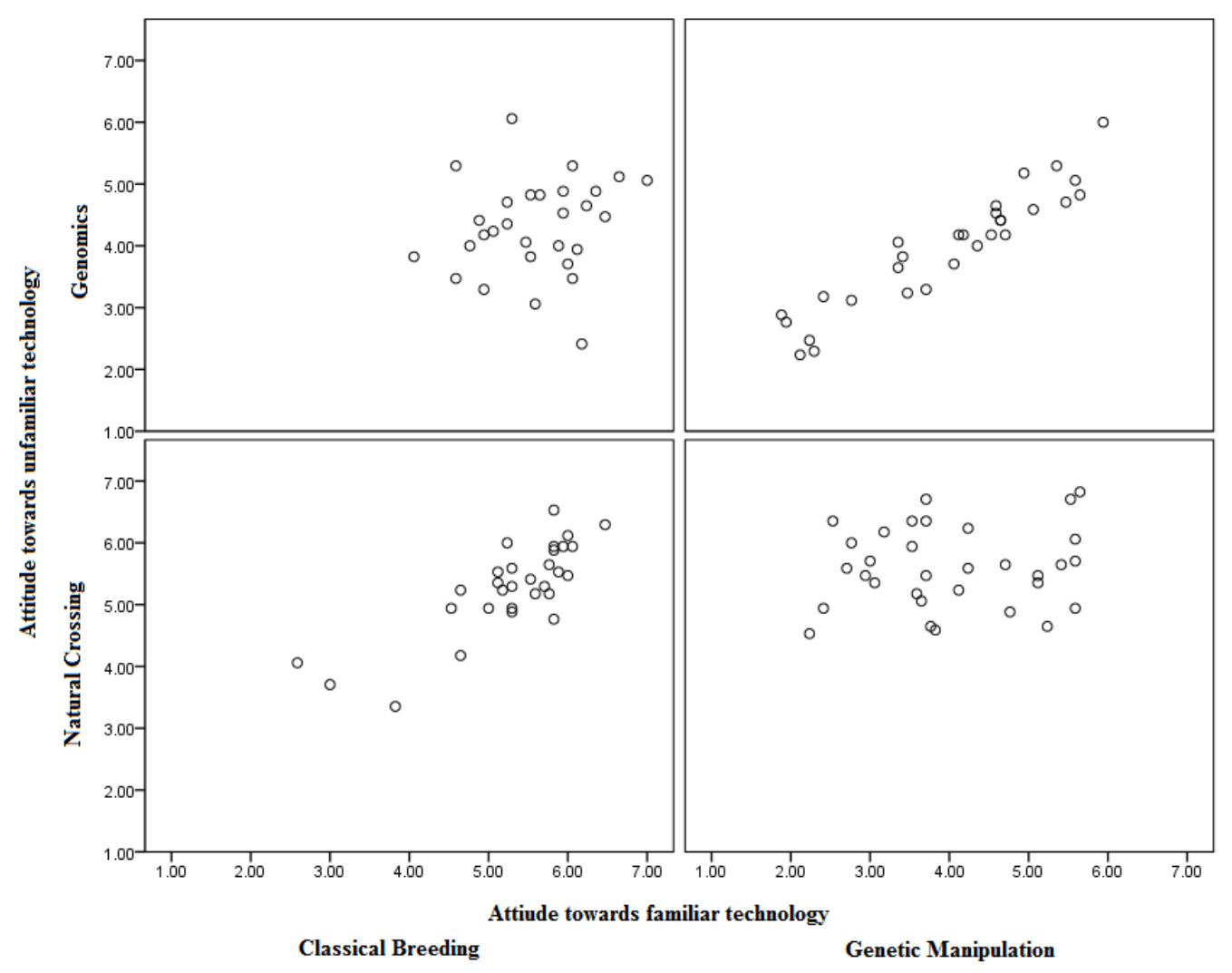

Figure 2. The relation between the attitudes towards resp. Classical Breeding and Genetic Manipulation and the initial attitudes towards resp. Genomics and Natural Crossing.

\section{Comparison effects}

Whereas attitude extension is concerned about the source of the attitude, comparison effect relates the extent to which the attitude extension is influenced by the context. With respect to comparison effects, the main question is the extent to which the evaluation of the unfamiliar is influenced by the technologies after which it is presented. The presumption is that the attitudes towards the familiar technology presented in the context (GM or TB, respectively) are stable and have an influence on the attitude towards the unfamiliar technology. To exclude the possibility of the attitudes towards GM or TB being influenced by the unfamiliar technology, we apply an ANOVA to see if there are significant differences in evaluation of GM or TB depending on the unfamiliar technology with which it is presented. A 2 (context) $\times 2$ (name technology) ANOVA of the average context scores revealed only a significant main effect of context, $F(1,116)=61.39, p<.001$, $\eta_{\mathrm{p}}^{2}=.35$, indicating that the evaluation of the familiar technology was not influenced by the unfamiliar technology with which it was presented. Genetic manipulation was regarded more negatively, $M=4.00, S D=1.13$, than traditional breeding, $M=5.43, S D=0.80$. These average evaluations of the familiar technologies enable us to study the direction of the comparison effects.

We expected that, in the conditions where genetic manipulation was presented as the context technology, the emerging attitudes about genomics would assimilate in the direction of genetic manipulation, and the emerging attitudes about natural 
crossing would contrast away from genetic manipulation. A 2 (context) $\times 2$ (name technology) ANOVA (see Figure 3) revealed a main effect due to name, $F(1,116)=$ $84.62, p<.001, \eta_{\mathrm{p}}^{2}=.42$. The name genomics caused a more negative attitude than the name natural crossing. Also, a significant interaction effect was observed, $F(1$, $116)=5.46, p<.05, \eta_{\mathrm{p}}^{2}=.05$, showing that the resulting attitudes were influences by the context. Simple effect analysis showed a trend in the difference in appreciation for genomics between the different contexts, with genomics being regarded as less negative when it was presented in a context with traditional breeding, $M=4.30, S D$ $=0.77$, than when it was presented in a genetic manipulation context, $M=3.97, S D$ $=0.96, F(1,116)=2.72, p=.10$. When these attitudes scores are compared to the attitude scores for traditional breeding and genetic manipulation, we see that the attitude score of genomics is nearly identical to that of genetic manipulation when they are presented together. When genomics was presented in combination with traditional breeding, genomics was evaluated more favourably than when it was presented in combination with genetic manipulation. As traditional breeding is evaluated more favourably than genetic manipulation, we can conclude that the evaluation of genomics assimilated towards the evaluation of traditional breeding. Hypothesis 2a can thus not be confirmed, since we expected a contrast effect. Nevertheless, although in a different direction, comparison effects did occur.

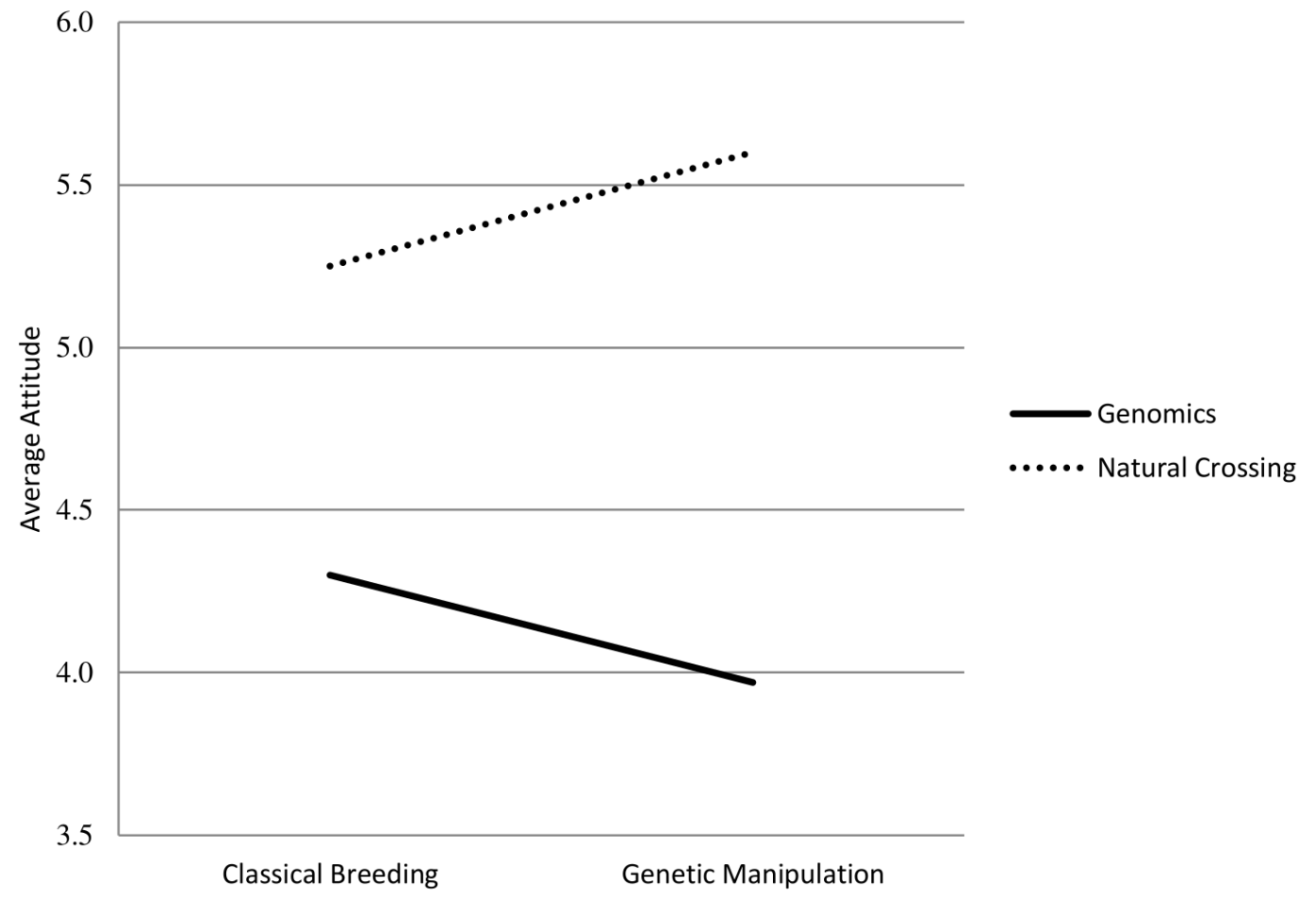

Figure 3. The average attitudes towards resp. Genomics and Natural Crossing when being presented in combination with resp. Classical Breeding and Genetic Manipulation.

When the name natural crossing was used, the opposite effect was observed, with a better appreciation when the name was presented in the context with genetic manipulation, $M=5.60, S D=0.65$, than in the traditional breeding context, $M=$ $5.25, S D=0.73, F(1,116)=3.46, p=.065$. Genetic manipulation was evaluated less favourably than traditional breeding, yet the evaluation of natural crossing was higher when it was presented with genetic manipulation than when it is presented 
with traditional breeding. Therefore, confirming Hypothesis $2 b$, we can conclude that the evaluation of natural crossing contrasts away from the evaluation of genetic manipulation when both technologies are presented together.

General discussion
So far, little attention has been given to the effects that a name of a (food production) technology can have on its interpretation. Most research on technologies investigates the way people react to a technology after they receive information [Bos, Koolstra and Willems, 2009]. However, as indicated by the experiences of experts working on food production technologies, it appears that the beliefs and attitudes about a technology may be influenced by the name alone, without additional information. In the current paper, we have investigated the effects of a technology's name on emerging attitudes, using genomics as a case. Building on categorization theory, we expected that the emerging attitudes of a new technology and related food products could be predicted by identifying attitudes about a familiar technology with a similar name. In addition, we expected comparison effects due to the combination of the name given to a technology and the presentation context. Further, we investigated the possible moderating role of need for cognitive closure on attitude extension.

The current study has shown that the categorization of an unfamiliar technology can determine the attitude towards that technology. Reported attitudes about genomics were virtually identical to the reported attitudes about GM, and the reported attitudes about natural crossing were very close to the reported attitudes about traditional breeding. The answers to the open question show that this is not due to knowledge about the unfamiliar technology, as the vast majority of respondents indicated that they were not familiar with genomics or natural crossing. Therefore it can be concluded that the attitudes towards the unfamiliar technologies are formed through attitude extension.

In addition, comparison effects had an influence on the evaluations. The evaluations of both genomics and natural crossing were influenced by the technology with which they were presented, although in a different direction. Genomics was evaluated more favourably when it was presented with traditional breeding than when it was presented with genetic manipulation. Natural crossing, on the other hand, was evaluated more favourably when it was presented with genetic manipulation. Several conclusions can be drawn from the observed patterns.

When comparing the effect of changing the familiar context technology with the effects of the name of the technology, it can be concluded that the name influences perceptions more than the familiar technology presented in the context. Whereas the name natural crossing led to systematically more favourable attitudes and more acceptance than the name genomics, the differences in evaluations caused by the familiar technology caused only minor variations. We can therefore conclude that the differences in evaluations are primarily caused by categorization and extension, with comparison effects influencing the attitudes only to a small extent.

The results show that genomics is evaluated more favourably when presented in a context with traditional breeding. This finding was unexpected, since we expected that genomics would be evaluated more favourably when presented in combination with genetic manipulation. We expected contrast to prevail due to the 
reported confusion between GM and genomics. We presumed that, if GM and genomics were regarded as being the same, it would logically follow that the comparison between traditional breeding and genomics would lead to contrast. However, an important determinant of comparison effects is the ambiguity experienced towards the concepts of evaluation [Higgins, 1989]. The prevalence of assimilation points to the possibility that, even though participants report very similar attitudes towards GM and genomics, they might experience uncertainty about whether they are identical. This uncertainty leaves room for the influence of the presentation context to give meaning, rather than to cause comparison. In general, we would recommend testing the effects of different familiar technologies that could possibly be used to explain the interpretation of an unfamiliar technology. With respect to explaining the current genomics case, the result shows that more favourable attitudes to genomics might be attained not by explaining its difference from GM, but by explaining how it relates to traditional breeding. Unfortunately, this might be very difficult to achieve, because people automatically link genomics to GM by categorization and tend to bring GM into the discussion even when it is not present [Hall, 2010].

In the experiment, we forced participants to give an evaluation about technologies with which they were not familiar. The current experiment could be criticized for lacking an 'I don't know' option. The reason for not including such a response option is that, when someone is confronted with a food produced with an unknown technology in real life in a situation that forces an evaluation, there is no escape from making the evaluation. For example, someone might be forced to choose between a regular and a genomics tomato during purchasing at a supermarket. This research shows that, in such situations, people can form an evaluation. With all participants showing the same strategy, it can be concluded that the evaluation was more than just a random guess, different from person to person and therefore unpredictable. From the current results, it appears to be a universal strategy that can be predicted.

Critics might argue that people who believe genomics and genetic modification to be strongly associated are actually right. There are many features that make it viable to cluster the two together, such as using information about genes for breeding and issues with intellectual properties. Although these are valid points, categorizing the technologies together on the basis of these attributes requires knowledge about both. The majority of the respondents explicitly stated that they associated them purely by name, and, with the exception of one, no respondent demonstrated detailed knowledge. In addition, it has been found that public knowledge about GM is generally very low [Frewer, Shepherd and Sparks, 1994]. More importantly, there are a number of attributes that genomics and traditional breeding share that makes it viable to categorize them together. The point is that, when people do not know anything about a technology, the name will determine the categorization, and the name will be the primary element in determining which category will be used when there are several potential categories. The category used then determines the evaluation of the technology and food produced using that technology [Ferguson and Bargh, 2004]. 


\section{Implications}

This research has several implications. First, at development stage of new technologies, experts should understand the full impact that the categorization of a new technology can have on its acceptance. This study shows that a name can play a crucial role in the categorization process and is therefore not a just meaningless characteristic. Rather, it is a label which steers interpretations and which is an important - in some cases even the primary — element in shaping initial attitudes towards new food technologies. Experts tend to choose names that are only meaningful for people who have the necessary expertise. Unfortunately, without this expertise, a name can trigger categorization and subsequently beliefs and attitudes that are unexpected or even believed to be improper by experts.

Therefore, we recommend that experts should focus on developing names that can aid in giving a more proper interpretation even if the name will have to be processed without expert knowledge.

When selecting a name, it can be beneficial to approach the name as a communication frame. Approached from a cognitive perspective, a frame can be defined as basic cognitive structures which guide the perception and representation of reality [Koenig, 2004]. The framing of information influences the assignment of the presented information to cognitive categories [Carter, 2013; Gitlin, 2003]. Often times in communication, presented frames are phrases, stories or images that activate such categories. However, the current research shows that a name can effectively act as a frame by itself [see also Bolsen, Palm and Kingsland, 2019], and even do so when there is no additional external information provided. As such, it is not so much external information that is being framed and processed, but the process of trying to find an answer to the question "what is it?".

This study shows the importance of a name that is understood by the public. This can advance lay understanding in both situations where people make uninformed (consumer) decisions or meet with experts in debates. To achieve mutual meaningful and understandable names as much as possible, it move the ownership of the process from solely the expert community and engage in a process of co-creation a name together with the public.

\section{Conclusion}

In this experiment, we tested the extent to which people are influenced by the name of a food production technology alone. The main result is that participants unanimously use their attitudes about a familiar technology (GM) to formulate answers about the unfamiliar technology (genomics). The extent to which these new attitudes can affect the interpretation of new information is an important topic for further research. If the new formed attitudes have an effect on the interpretation of new information, the consequences of a name might even go beyond the current findings. With respect to the current experiment, it can safely be concluded that a name of a technology can influence the success of its introduction and therefore deserves careful planning. Just because of its name, people might refuse to accept genomics and foods produced with the technology since they believe genomics to be similar to GM. 
Aron, A., Aron, E. N. and Smollan, D. (1992). 'Inclusion of other in the self scale and the structure of interpersonal closeness'. Journal of Personality and Social Psychology 63 (4), pp. 596-612. https://doi.org/10.1037/0022-3514.63.4.596.

Bauer, M., Durant, J. and Gaskell, G. (1998). 'Biology in the public sphere: a comparative review'. In: Biotechnology in the public sphere. Ed. by J. Durant, M. Bauer and G. Gaskell. London, U.K.: Science Museum, pp. 217-227.

Boersma, R. and Gremmen, B. (2018). 'Genomics? That is probably GM! The impact a name can have on the interpretation of a technology'. Life Sciences, Society and Policy 14 (1). https: //doi.org/10.1186/s40504-018-0072-3.

Bolsen, T., Palm, R. and Kingsland, J. T. (2019). ‘Counteracting climate science politicization with effective frames and imagery'. Science Communication 41 (2), pp. 147-171. https://doi.org/10.1177/1075547019834565.

Bos, M. J. W., Koolstra, C. M. and Willems, J. T. J. M. (2009). 'Adolescent responses toward a new technology: first associations, information seeking and affective responses to ecogenomics'. Public Understanding of Science 18 (2), pp. 243-253. https://doi.org/10.1177/0963662507087306.

Brunel, M., Launay, C., Le Floch, V., Py, J., Cascino, N., Zorapapillan, M. and Lo Monaco, G. (2017). 'Is the social representation of nanotechnology anchored in that of GMOs?' Journal of Risk Research 21 (10), pp. 1248-1263. https://doi.org/10.1080/13669877.2017.1304976.

Carter, M. J. (2013). 'The hermeneutics of frames and framing: an examination of the media's construction of reality'. SAGE Open 3 (2), p. 215824401348791. https://doi.org/10.1177/2158244013487915.

Ferguson, M. J. and Bargh, J. A. (2004). 'How social perception can automatically influence behavior'. Trends in Cognitive Sciences 8 (1), pp. 33-39. https://doi.org/10.1016/j.tics.2003.11.004.

Frewer, L. J., Shepherd, R. and Sparks, P. (1994). 'Biotechnology and food production: knowledge and perceived risk'. British Food Journal 96 (9), pp. 26-32. https://doi.org/10.1108/00070709410072562.

Friedrich, B. (6th September 2016). 'Clean meat: the "clean energy" of food'. Huffington Post. URL: http://www . huffingtonpost. com/entry/clean-meat-the -clean-energy-of-food_us_57cee00ee4b0f831f705a879.

Gitlin, T. (2003). The whole world is watching: mass media in the making and unmaking of the new left. Berkeley, CA, U.S.A.: University of California Press.

Gregan-Paxton, J. (2001). 'The role of abstract and specific knowledge in the formation of product judgments: an analogical learning perspective'. Journal of Consumer Psychology 10 (3), pp. 141-158. https://doi.org/10.1207/s15327663jcp1003_01.

Gregan-Paxton, J., Hoeffler, S. and Zhao, M. (2005). 'When categorization is ambiguous: factors that facilitate the use of a multiple category inference strategy'. Journal of Consumer Psychology 15 (2), pp. 127-140. https://doi.org/10.1207/s15327663jcp1502_5.

Gregan-Paxton, J. and John, D. R. (1997). 'Consumer learning by analogy: a model of internal knowledge transfer'. Journal of Consumer Research 24 (3), pp. 266-284. https://doi.org/10.1086/209509.

Hall, R. (2010). 'CBSG2012: a public-private partnership in the plant sciences'. In: CSG researchers days 2010. Symposium organized at the meeting of CSG (CSG Centre for Society and the Life Sciences, Berg en dal, The Netherlands).

Hansen, A. (2010). Environment, media and communication. London and New York: Routledge. 
Herr, P. M. (1986). 'Consequences of priming: judgment and behavior'. Journal of Personality and Social Psychology 51 (6), pp. 1106-1115. https://doi.org/10.1037/0022-3514.51.6.1106.

Higgins, E. T. (1989). 'Knowledge accessibility and activation: subjectivity and suffering from unconscious sources'. In: Unintended thought. Ed. by J. S. Uleman and J. A. Bargh. New York, NY, U.S.A.: Guilford Press, pp. 75-123.

Ingold, T. and Kurttila, T. (2000). 'Perceiving the environment in finnish lapland'. Body E Society 6 (3-4), pp. 183-196. https://doi.org/10.1177/1357034x00006003010.

Kampers, F. (2009). What nanotechnology can do for your average donut. URL: http://2020science.org/2009/03/30/what-nanotechnology-can-do-fo $r$-your-average-donut/ (visited on 29th August 2017).

Koenig, T. (2004). Routinizing frame analysis through the use of CAQDAS. URL: http://www.restore.ac.uk/lboro/research/case_studies/hohmann/fr ames_and_CAQDAS.pdf (visited on 17th May 2019).

Loken, B., Barsalou, L. W. and Joiner, C. (2008). 'Categorization theory and research in consumer psychology: category representation and category-based inference'. In: Handbook of consumer psychology. Ed. by C. P. Haugtvedt, P. M. Herr and F. R. Kardes. New York, NY, U.S.A.: Lawrence Erlbaum Associates, pp. 133-163.

Mussweiler, T., Michels, C. and Weiss, A. (2017). 'Reflections on comparison'. In: Reflective and impulsive determinants of human behavior. Ed. by R. Deutsch, B. Gawronski and W. Hofmann. New York, NY, U.S.A.: Routledge, pp. 19-34.

Muthukrishnan, A. V. and Weitz, B. A. (1991). 'Role of product knowledge in evaluation of brand extension'. In: Advances in consumer research. Ed. by R. H. Holman and M. R. Solomon. Vol. 18. Provo, UT, U.S.A.: Association for Consumer Research, pp. 407-413.

Rosch, E. (1978). 'Principles of categorization'. In: Cognition and categorization. Ed. by E. Rosch and B. B. Loyd. Hillsdale, NJ, U.S.A.: Lawrence Erlbaum, pp. 27-48.

Stoner, J. L., Loken, B. and Stadler Blank, A. (2018). 'The name game: how naming products increases psychological ownership and subsequent consumer evaluations'. Journal of Consumer Psychology 28 (1), pp. 130-137. https://doi.org/10.1002/jcpy.1005.

Van Dam, F. and De Vriend, H. (2002). Publieksonderzoek Genomics 2002. Den Haag, The Netherlands: Stichting Consument en Biotechnologie.

Van den Heuvel, T., Renes, R. J., Van Trijp, H., Gremmen, B. and Van Woerkum, C. (2008). 'Consumer judgment regarding genomics: exploring the influence of initial categorization and different modes of thought'. In: Etmaal van de Commuicatiewetenschap. Amsterdam, The Netherlands: Vrije Universieteit.

Reginald Boersma is a cognitive psychologist who completed his $\mathrm{PhD}$ studies at the philosophy and the strategic communication groups of Wageningen University. He is interested in how people use and interact with technology and how they decide which technologies they adopt or reject. His studies focus on the way people make decisions in situations they lack related expertise and use ques instead. Wageningen University, Philosophy, Hollandseweg 1, Bode 90, $6706 \mathrm{KN}$, Wageningen, the Netherlands. T. +31 (0)317 484310 .

E-mail: Reginald.Boersma@gmail.com. 
P. Marijn Poortvliet is an associate professor and studies how psychological factors interact with risk communication. Second, he investigates behavioral and communicative factors that explain the adoption of technologies and practices. Finally, he studies how behavioral and communicative factors in uptake of practices interact with other and higher level factors, such as the systemic features in which practices are embedded. Together, these lines of research address the communicative interaction between humans and life science issues defined by risk and uncertainty. Wageningen University, Strategic Communication, Hollandseweg 1, Bode 79, 6706 KN, Wageningen, the Netherlands. T. +31 (0)317 484004. E-mail: Marijn.Poortvliet@wur.com.

Bart Gremmen is professor of ethics in life sciences. He is known for applying ethical theory to life science domains with a strong focus on plant, animal and environmental ethics. His collaboration with different Wageningen UR ensures a multi-disciplinary approach. He has coordinated three large NWO genomics programs. He is member of the management team of the 4TU Ethics, and has established a strong international network of environmental and applied ethicists. He is also chair of the Ethics working group of the European Association of Animals Scientist. Wageningen University, Philosophy, Hollandseweg 1, Bode 90, $6706 \mathrm{KN}$, Wageningen, the Netherlands. T. +31 (0)317 483810.

E-mail: Bart.Gremmen@wur.com.

\section{How to cite}

Boersma, R., Poorvliet, P. M. and Gremmen, B. (2019). 'Naming is framing: the effects of a technological name on the interpretation of a technology'. JCOM 18 (06), A04. https:/ /doi.org/10.22323/2.18060204. 